\title{
MAGNETIZAÇÃO REMANESCENTE: UM PARÂMETRO CRUCIAL PARA A INTERPRETAÇÃO E MODELAMENTO DE ANOMALIAS MAGNÉTICAS EM TERRITÓRIO BRASILEIRO
}

\author{
Renato Cordani ${ }^{1}$ e Wladimir Shukowsky² \\ Recebido em 6 agosto, 2009 / Aceito em 5 janeiro, 2010 \\ Received on August 6, 2009 / Accepted on January 5, 2010
}

\begin{abstract}
Remanent magnetization is often neglected in data interpretation, probably for the difficulty on working with it. In most of the cases, both in academic works and professional models in mining and oil industry, prevails the assumption that remanence is irrelevant or inexistent, and the induced effect is the only one considered. The present paper shows that using this parameter is particularly important on Brazilian anomalies, where magnetic latitudes are low, and try to provide tools for the use of the remanence information. In the article we discuss the use of common geophysical techniques, analytic signal and reduction to the pole in Brazilian anomalies due to rocks with and without remanence. Then we presented a method to estimate the total magnetic direction from a magnetic anomaly, from which it is possible to extract the total magnetization parameter, and consequently the resultant sum vector after induced and remanent parameters. Finally, we present a methodology for the use of remanent information to determine the age of the source rocks.
\end{abstract}

Keywords: remanent magnetization, magnetic anomalies, interpretation, paleomagnetic virtual poles.

RESUMO. A questão da magnetização remanescente na interpretação de anomalias magnéticas é frequentemente negligenciada, principalmente em função da dificuldade em se lidar com a mesma. Na maioria dos casos, tanto nos trabalhos acadêmicos quanto nos modelos que circulam nos meios profissionais da exploração mineral e de petróleo, assume-se que a magnetização remanescente é desprezível e utiliza-se apenas a induzida. 0 presente artigo mostra que 0 uso desse parâmetro é particularmente importante no tocante às anomalias magnéticas brasileiras, e procura fornecer subsídios para 0 uso desta informação. Discute-se 0 uso de duas técnicas consagradas, a Redução ao Pólo e o Sinal Analítico, em anomalias brasileiras com e sem magnetização remanescente. Mostramos a aplicação da técnica de determinação da magnetização total, permitindo que os modelos sejam construídos a partir da resultante da soma das magnetizações induzida e remanescente, e posteriormente apresentamos uma metodologia de uso da informação remanescente na datação das rochas fonte.

Palavras-chave: magnetização remanescente, anomalias magnéticas, interpretação, pólos paleomagnéticos virtuais.

\footnotetext{
${ }_{1}$ Reconsult Geofísica, Rua Dr. Guilherme Bannitz, 126, conj. 92, 04532-060 São Paulo, SP, Brasil. Tel.: (11) 3845-1842; Fax: (11) 3845-1842 - E-mail: rcordani@reconsult.com.br

2IAG-USP, Rua do Matão, 1226, Cidade Universitária, 05508-090 São Paulo, SP, Brasil. Tel.: (11) 3091-4762; Fax: (11) 3091-2801 - E-mail: wladimir@iag.usp.br
} 


\section{INTRODUÇÃo}

A componente remanescente das anomalias magnéticas tem afligido os geofísicos há bastante tempo, e ainda está longe de ser resolvida a contento, haja vista os artigos referentes ao assunto que continuam a aparecer ao longo dos últimos 30 anos, e.g. Shurbet et al. (1976), Schnetzler \& Taylor (1984), Roest \& Pilkington (1993), McEnroe \& Brown (2000), Thurston (2001).

Na comparação com o outro método potencial, a gravimetria, que tem apenas a densidade como propriedade física, a magnetometria é obrigada a trabalhar com duas propriedades físicas, a susceptibilidade e a magnetização remanescente. A magnetização induzida é função da posição da fonte magnética na Terra e portanto pode ser bem controlada. Por outro lado, a magnetização remanescente pode assumir virtualmente qualquer direção, sendo portanto um parâmetro muito difícil de estimar, consequentemente simplesmente "não usá-Io" tem sido a modalidade mais utilizada. Essa dificuldade para tratar a remanescência tem inclusive estimulado o desenvolvimento de técnicas de alta centricidade como o Sinal Analítico, muito popular no Brasil.

Particularmente no Brasil, onde a intensidade do campo magnético é menor em função da Anomalia do Atlântico Sul (Hartmann \& Pacca, 2009), a componente remanescente da magnetização torna-se relativamente mais importante frente à magnetização induzida.

A intenção do presente trabalho é fornecer técnicas de uso da informação sobre a magnetização remanescente para 0 modelamento e interpretação das anomalias brasileiras.

\section{Particularidades do campo magnético brasileiro}

A maior parte da literatura de magnetometria aplicada à exploração foi escrita no Canadá ou Austrália. Esses dois países são os que notadamente fazem uso de magnetometria aplicada à mineração e têm maior know how em aerolevantamentos magnéticos. Coincidência ou não, esses dois países têm no tempo presente um campo magnético bastante peculiar: ambos estão no topo da escala de intensidade do campo e adicionalmente são os países mais próximos dos pólos magnéticos.

Comparativamente, 0 campo magnético brasileiro é também peculiar, pois é cerca de três vezes menor. Para a anomalia causada por uma rocha, considerando apenas o campo devido à magnetização induzida, temos:

$$
\mathbf{M}=k \mathbf{H},
$$

onde $k$ é a susceptibilidade da rocha (escalar), $\mathbf{H}$ é 0 vetor intensidade magnética da Terra naquele ponto e $\mathbf{M}$ é 0 vetor magnetização.
Como 0 campo devido à magnetização induzida é linear em relação à magnetização $\mathbf{M}$, uma mesma rocha de susceptibilidade $k$ vai causar no Canadá ou Austrália uma anomalia com amplitude de duas a três vezes maior do que no Brasil. Exemplificando, digamos que uma determinada chaminé vulcânica com uma dada susceptibilidade $k$ e com magnetização remanescente desprezível, gera no Brasil uma anomalia magnética de $60 \mathrm{nT}$. No Canadá, a mesma chaminé geraria uma anomalia de cerca 160 nT. Constata-se assim que no Brasil os campos anômalos causados pela magnetização induzida são muito mais fracos do que no Canadá e Austrália.

Além de mais fracos, há uma dificuldade adicional: ao contrário do Canadá e Austrália, que estão situados próximo aos pólos magnéticos, onde a inclinação magnética é em torno de $70^{\circ}, 0$ Brasil situa-se próximo ao equador geomagnético, onde a inclinação magnética é baixa. Próximo aos pólos magnéticos, as anomalias geradas por um contraste positivo de susceptibilidade geram um "alto" no campo magnético, enquanto que próximo ao equador geomagnético as anomalias são fundamentalmente "baixos" no campo.

A Figura 1 a seguir mostra 0 perfil da anomalia magnética calculada a partir de um cilindro magnético no Brasil, no Canadá e na Austrália (considerando a magnetização remanescente desprezível):

Na observação da Figura 1, fica evidente que 0 campo magnético induzido no Brasil é muito mais fraco que nos outros dois países. Além disso, no Canadá o centro do corpo é mais ou menos coincidente com o pico da anomalia positiva, de forma análoga à gravimetria e ao senso comum. A anomalia australiana não está centralizada no corpo, mas a amplitude é mais de três vezes maior do que a brasileira, e o "alto" é mais proeminente que o "baixo". Na anomalia brasileira, o centro do corpo está entre o "baixo" e 0 "alto" da anomalia, sendo o "baixo" mais forte, e a anomalia é comparativamente fraca, dificultando a interpretação.

Se o campo magnético induzido é comparativamente menor, por conseguinte a componente da anomalia devida à magnetização remanescente é relativamente mais importante no Brasil. Desta forma, uma metodologia que seja capaz de não apenas contornar o "problema" da magnetização remanescente mas, ao contrário, aproveitá-la para obter informações para auxiliar a interpretação, é de suma importância na interpretação de anomalias em geral, e sobretudo no território brasileiro.

\section{As técnicas de alta centricidade}

Para contornar o "problema" da magnetização remanescente e auxiliar a interpretação, diversas técnicas geofísicas que contri- 


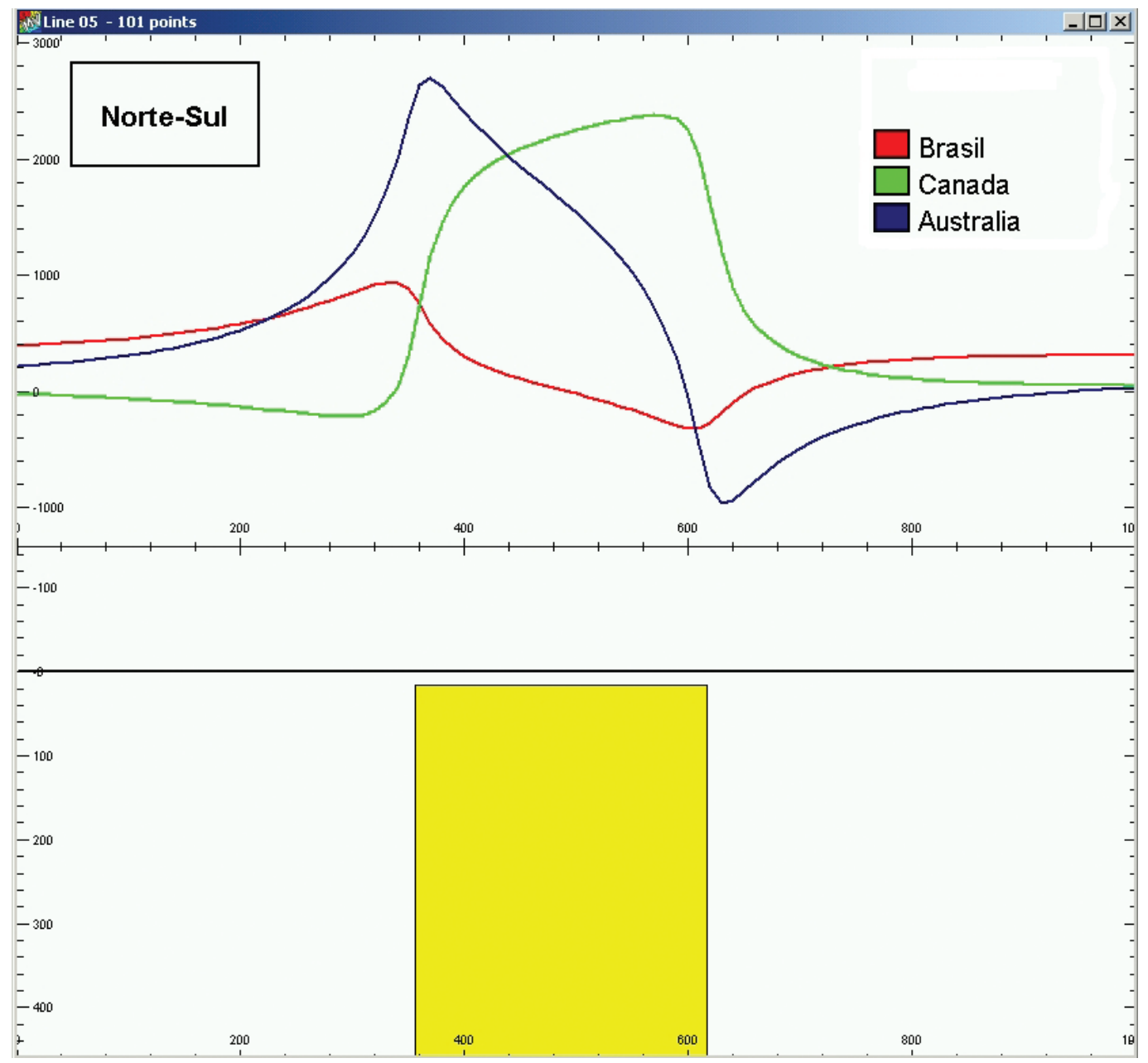

Figura 1 - Comparação entre anomalias induzidas por um mesmo corpo sintético no Brasil, Canadá e Austrália. Parâmetros: (Campo, I, D); Brasil $=(23.000,-20,-20)$; Canadá $(60.000,80,0)$; Austrália $(60.000,-60,5)$.

buem para "eliminar" o problema são costumeiramente aplicadas, sendo a Redução ao Pólo e o Sinal Analítico as mais populares.

A aplicação da Redução ao Pólo, cuja utilização em baixas latitudes magnéticas era desencorajada em função de instabilidade numérica, ganhou impulso nos últimos anos com 0 desenvolvimento de técnicas que a estabilizam, como por exemplo Keating \& Zerbo (1996), Rajagopalan (2003), Li (2008). A aplicação do Sinal Analítico a anomalias brasileiras é uma prática consagrada, sendo inclusive um produto padrão nas licitações do serviço geológico brasileiro (CPRM).

Ambas as técnicas são muito eficientes em simplificar a interpretação das anomalias magnéticas, pois além de "centrar" as anomalias relativamente à fonte, ainda transformam as anomalias em grandezas diretamente proporcionais à força destas, analogamente à gravimetria. Não obstante a aplicabilidade de tais técnicas, é necessário salientar que nenhuma delas utiliza a informação de magnetização remanescente. Ao contrário, a técnica de Redução ao Pólo assume que a remanescente não existe, enquanto que a técnica do Sinal Analítico não utiliza essa informação, por computar apenas as derivadas do campo.

Para exemplificar a aplicação das técnicas de alta centricidade citadas, apresentamos nas Figuras 2a e 3a as anomalias magnéticas de campo total dos complexos alcalinos de Tapira e Araxá, duas anomalias devidas a rochas de natureza e idade radiométrica similar, porém com naturezas magnéticas bastante diferentes, sendo Araxá semelhante a um dipolo normal e Tapira a um dipolo invertido.

As óbvias informaç̧ões adicionais que se podem tirar do fato de que as naturezas magnéticas entre os dois complexos são muito diferentes perdem-se ao se executar o Sinal Analítico, conforme as Figuras $2 b$ e $3 b$.

As Figuras $2 \mathrm{~b}$ e $3 \mathrm{~b}$ mostram que mesmo 0 complexo alca- 
lino de Tapira sendo majoritariamente remanescente e o de Araxá sendo majoritariamente induzido, os respectivos sinais analíticos são muito similares, e a informação referente a essa diferença na natureza magnética é perdida. Desta forma, entendemos que as técnicas de alta centricidade nem sempre devem ser aplicadas de forma automática, e algumas anomalias brasileiras devem ser interpretadas de outras formas, utilizando algumas outras técnicas que veremos a seguir.
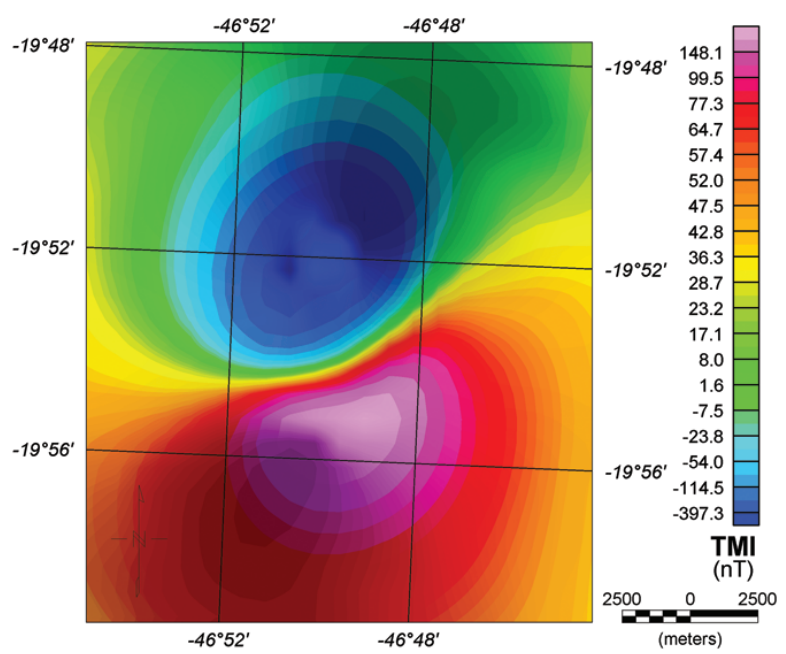

Figura 2(a) - Campo Magnético Total do complexo alcalino de Tapira.

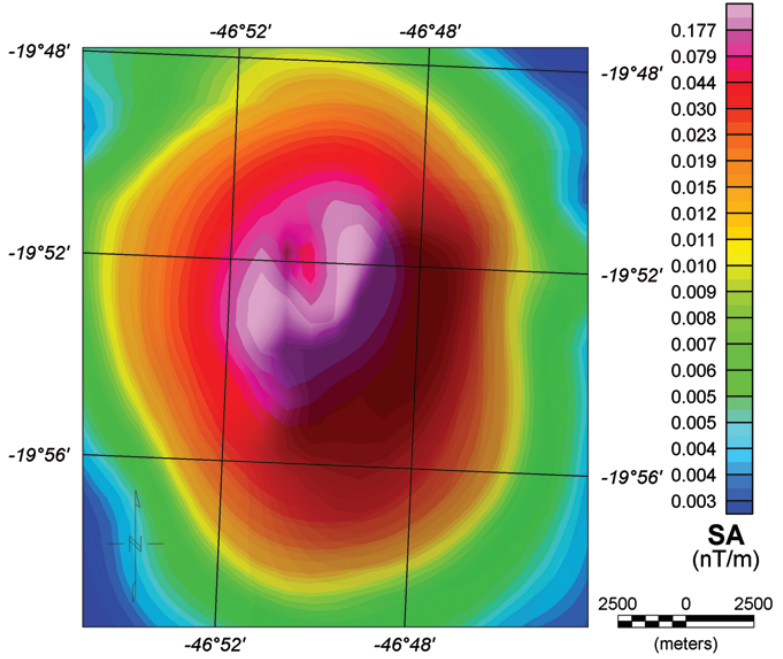

Figura 2(b) - Sinal Analítico do complexo alcalino de Tapira.

\section{Determinando a magnetização total}

Para usar a informação da magnetização remanescente, propomos que primeiro calcule-se a magnetização resultante total da anomalia (Induzida + Remanescente). Pode-se usar diversas metodologias para calcular a magnetização total de uma anomalia. 0 método que apresentamos a seguir é denominado MaxiMin, proposto e descrito por Fedi et al. (1994). 0 método é baseado em sucessivas aplicações do operador de redução ao pólo no campo magnético medido para diferentes parâmetros da inclinação e declinação da magnetização (I, D), escolhendo o par que maximiza o mínimo da anomalia magnética reduzida ao pólo. 0 resultado da aplicação do algoritmo é uma estimativa da direção da magnetização total da anomalia, ou seja, da resultante entre as magnetizações remanescente e induzida.

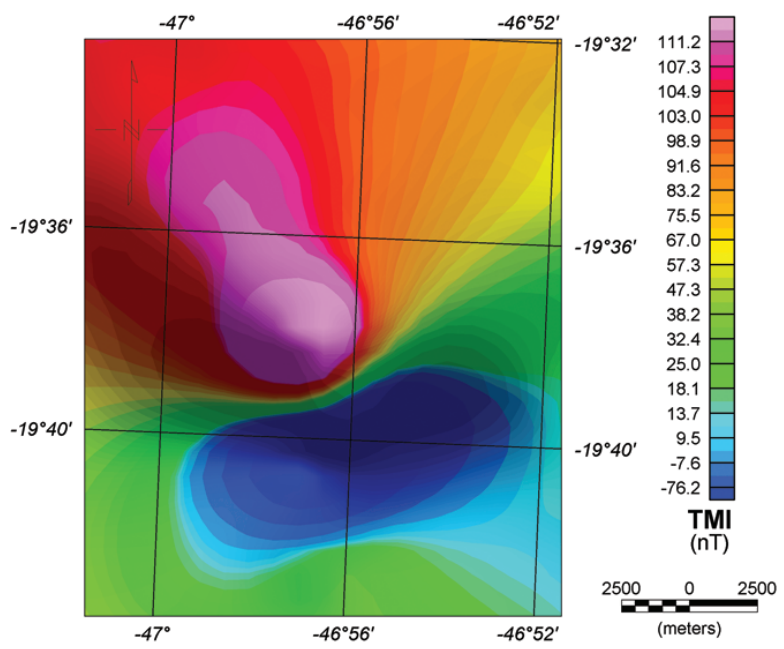

Figura 3(a) - Campo Magnético Total do complexo alcalino de Araxá.

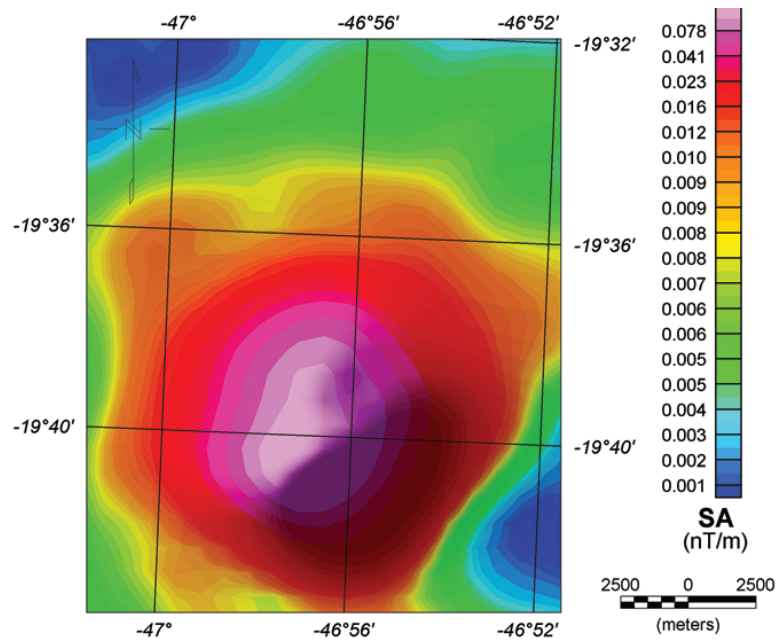

Figura 3(b) - Sinal Analítico do complexo alcalino de Araxá.

Para a anomalia de Tapira, cuja magnetização é majoritariamente remanescente, aplicamos a metodologia Maxi-Min e obtivemos os seguintes parâmetros (últimas duas colunas da Tab. 1).

$\mathrm{Na}$ tabela abaixo nota-se que se a direção de magnetização total da anomalia é muito diferente da direção do IGRF, razão pela qual é impossível, além de errôneo, modelar essa anomalia sem introduzir parâmetros de magnetização remanescente. Por outro lado, se introduzirmos o par $(I ; D)=(68,4 ;-174,1)$ como 
parâmetro de magnetização total 0 modelamento torna-se bastante fácil.

Tabela 1 - Localização, IGRF da época do levantamento e direção da magnetização total obtida com a aplicação do método Maxi-Min sobre a anomalia magnética do complexo alcalino de Tapira.

\begin{tabular}{|c|c|c|c|c|c|}
\hline \multicolumn{2}{|c|}{ Localização } & \multicolumn{2}{c|}{ IGRF } & \multicolumn{2}{c|}{ Magnetização total } \\
\hline Long & Lat & Incl & Decl & Incl & Decl \\
\hline$-19,89$ & $-46,83$ & $-19,40$ & $-16,20$ & 68,4 & $-174,1$ \\
\hline
\end{tabular}

É possível estimar o erro no cálculo Maxi-Min da magnetização total usando uma simulação numérica. Aplicamos o método Maxi-Min em anomalias provocadas por corpos magnetizados com direção fixa e variável considerando-se três configurações espaciais distintas, conforme a Figura 4.
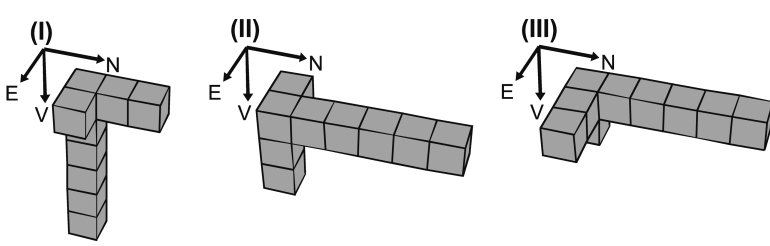

Figura 4 - Geometria dos modelos para simulação numérica da acuracidade do método Maxi-Min, cada um contendo nove cubos com 100m de lado. Consideramos três orientações espaciais (I, II e III).

Efetuamos a simulação 1200 vezes usando magnetização fixa (todos os cubos com a mesma magnetização) e variável (diferentes magnetizações para cada cubo). Para cada simulação comparamos a magnetização real dos blocos com a obtida através do método Maxi-Min, e mostramos a diferença nos diagramas da Figura 5.

As simulações numéricas fornecem uma estimativa de acurácia da ordem de 5,0 na determinação da magnetização total no método Maxi-Min com 95\% de intervalo de confiança.

\section{Usando a magnetização total para obter informações adicionais sobre a rocha fonte - a técnica VPMA}

Além das notórias vantagens de se aplicar a metodologia MaxiMin (ou qualquer metodologia similar) para o modelamento de anomalias magnéticas fazendo uso da direção de magnetização total em lugar da direção da magnetização induzida, pode-se procurar retirar informações da própria componente remanescente, conforme proposto por Cordani (2008) e Cordani \& Shukowsky (2009), em uma metodologia denominada VPMA ou Virtual Pole from Magnetic Anomaly.

0 cerne da metodologia VPMA consiste em isolar a componente remanescente de uma anomalia magnética. Para isso, primeiramente calcula-se a magnetização total da fonte magnética através do método Maxi-Min, e posteriormente retira-se a componente induzida, usando a seguinte equação vetorial:

$$
\vec{J}=\vec{J} i+\vec{J} r,
$$

onde $\vec{J}$ é a magnetização total da rocha, $\vec{J} i$ é a componente induzida e $\vec{J} r$ é a componente remanescente.

$\mathrm{Na}$ Equação [2], sabemos a direção do vetor $\vec{J} i$, pois é dado pelo IGRF, porém não sabemos a magnitude desse vetor. É possível saber sua magnitude quando conhecemos $Q$, a constante de Koenigsberger. Portanto, para retirar a componente induzida, é necessário determinar o valor de $Q$, que determina a relação escalar entre $\vec{J} i$ e $\vec{J} r$, segundo a seguinte equação:

$$
Q=J r / k * J i,
$$

onde $k$ é susceptibilidade magnética da rocha.

Nesta relação, quando a componente induzida ( $\vec{J} i$ ) é predominante, $Q$ é muito pequeno, e por outro lado, se a componente remanescente $(\vec{J} i)$ for predominante, os valores de $Q$ são mais altos. Portanto, para resolver a Equação [2] é necessário fornecer um valor de $Q$. Entretanto, em geral não é possível determinar 0 valor de $Q$, ou seja, embora saibamos o valor estimado para a magnetização total, não sabemos quanto da anomalia total é causado por indução $(\vec{J} i)$ e quanto é causado por remanescência.

A solução que propomos para esse impasse em arbitrar o vaIor de $Q$ é não optar por apenas um valor escolhido de $Q$, mas considerar todos os valores de $Q$ possíveis, desde o mínimo $Q$ até 0 máximo $Q$, desde 0 até infinito.

0 mínimo $Q$ teoricamente poderia ser zero, ou seja, toda a anomalia seria devida à indução pura. Porém, uma vez que seja calculada a magnetização total e a direção não coincida exatamente com o IGRF, há um valor mínimo de $Q$ necessário para satisfazer a Equação [2]. Já o valor máximo de $Q$ é aquele no qual $\vec{J} r$ converge para $\vec{J}$.

Posteriormente, calculamos o paleopólo correspondente a cada valor de $\vec{J} r$ encontrado, e por último comparamos as retas obtidas com a curva de deriva polar aparente (APWP - Apparent Polar Wander Path) para a placa tectônica em questão.

Aplicamos a metodologia VPMA descrita acima em quatro complexos alcalinos brasileiros: Tapira, Araxá, Juquiá e Pariqueraçu, e adicionalmente em uma anomalia magnética cuja fonte é desconhecida, denominada anomalia magnética de Registro. A localização dos complexos e as três anomalias magnéticas de campo total de Pariqueraçu, Juquiá e Registro estão nas Figuras 6 a 9 .

Os resultados da aplicação da metodologia VPMA são mostrados na Figura 10, confrontados com a curva de deriva polar 


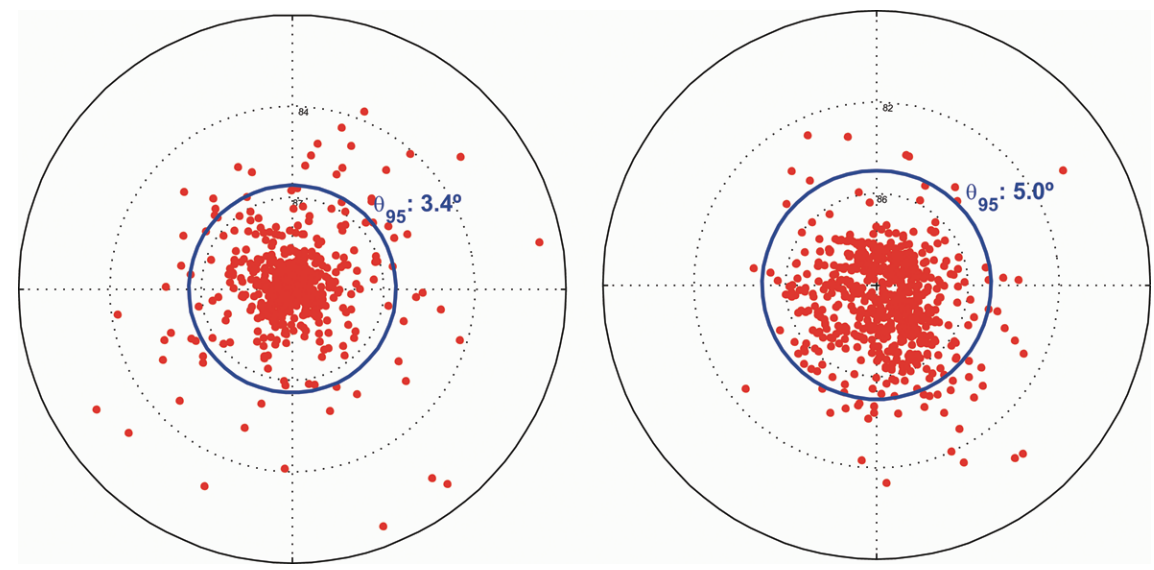

Figura 5 - Erro na estimativa da direção de magnetização usando 0 método Maxi-Min. 0 círculo azul determina 0 intervalo de confiança de 95\%. À esquerda, magnetização fixa; à direita, magnetização variável.

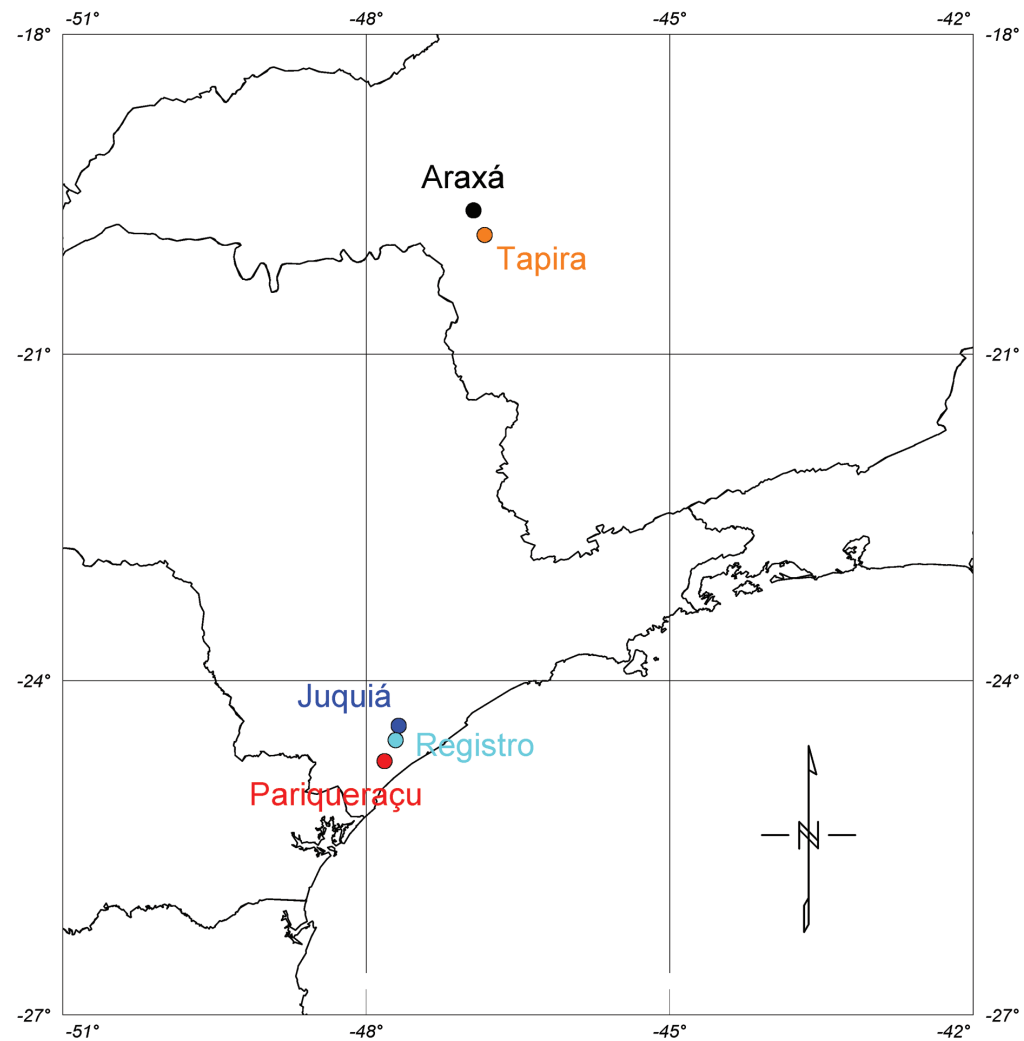

Figura 6 - Localização das anomalias perante os estados de São Paulo e Minas Gerais, Brasil.

aparente de Ernesto (2007). Em vista dos resultados, podem ser efetuados os comentários genéricos seguintes:

- Juquiá: com $Q=3$ recuperamos a idade de 130 Ma, que é a idade preferencial medida por geocronologia de acordo com Amaral et al. (1967).
- Registro: com $Q=0,88$ recuperamos a idade de $95 \mathrm{Ma}$. A rocha fonte dessa anomalia ainda não foi encontrada.

- Pariqueraçu: com $Q=10$ recuperamos a idade de $125 \mathrm{Ma}$. A rocha fonte dessa anomalia ainda não foi datada, porém Morbidelli et al. (2000), com base em dados geológicos e petrológicos sugerem que a idade da intrusão deverá ser 


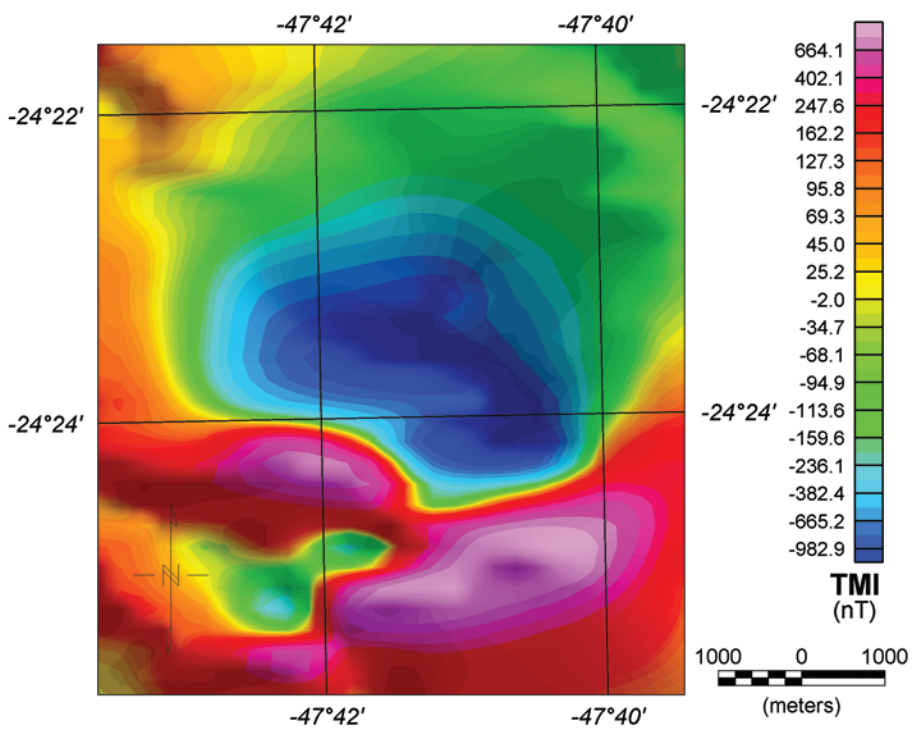

Figura 7 - Campo Magnético Total do complexo alcalino de Juquiá.

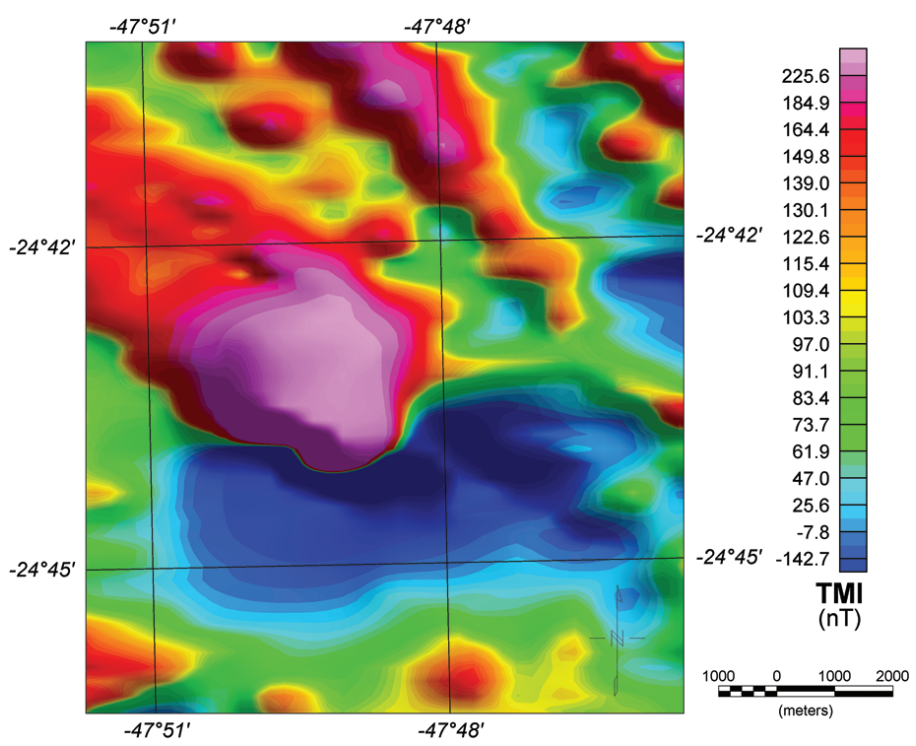

Figura 8 - Campo Magnético Total do complexo alcalino de Pariqueraçu.

próxima a $130 \pm 5 \mathrm{Ma}$, ou seja, corroboramos a sugestão de Morbidelli et al. (2000).

- Araxá: com $Q=0,35$ recuperamos a idade de $70 \mathrm{Ma}$, dentro da margem de erro da idade radiométrica medida que é de $80 \pm 10 \mathrm{Ma}$, de acordo com Gomes et al. (1990).

- Tapira: com $Q=2$ recuperamos a idade de $70 \mathrm{Ma}$, praticamente dentro da margem de erro da idade radiométrica medida que é de $79 \pm 8 \mathrm{Ma}$, de acordo com Hasui \& Cordani (1968).

\section{CONCLUSÕES}

Mostramos que no Brasil a magnetização remanescente é proporcionalmente mais importante do que na maioria dos outros países, e que as técnicas de alta centricidade (Redução ao Pólo e Sinal Analítico) nem sempre são as mais adequadas para a interpretação das anomalias magnéticas brasileiras.

Mostramos que 0 cálculo da magnetização total da anomalia combinado com a retirada da componente induzida através da metodologia VPMA - Virtual Pole from Magnetic Anomaly foi capaz de estimar com uma boa aproximação a idade de três com- 


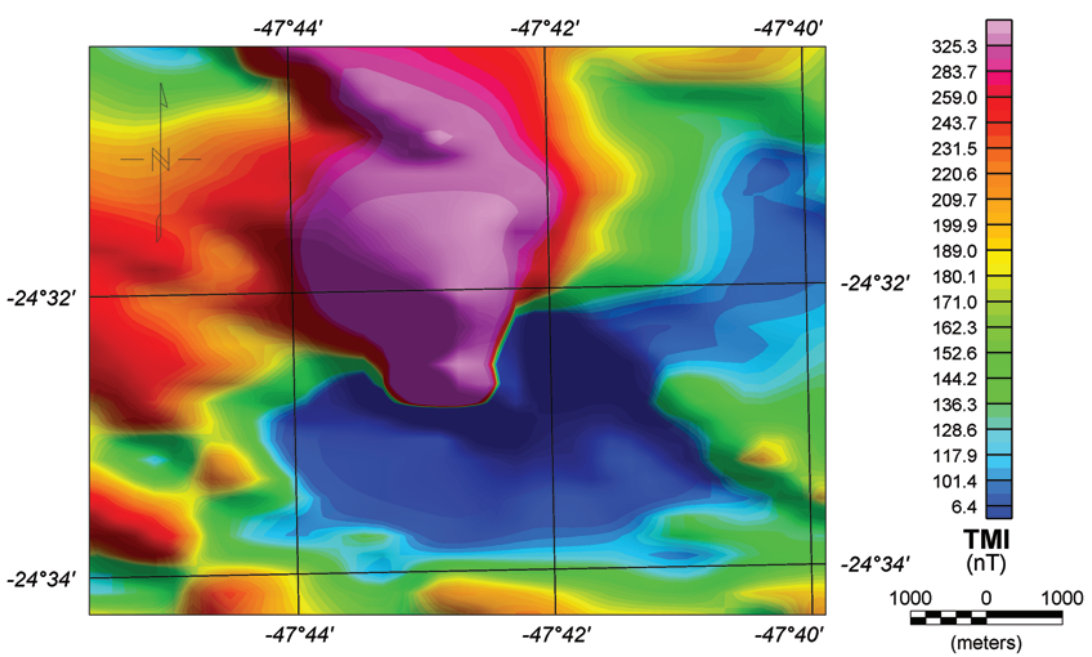

Figura 9 - Campo Magnético Total da anomalia magnética de Registro.

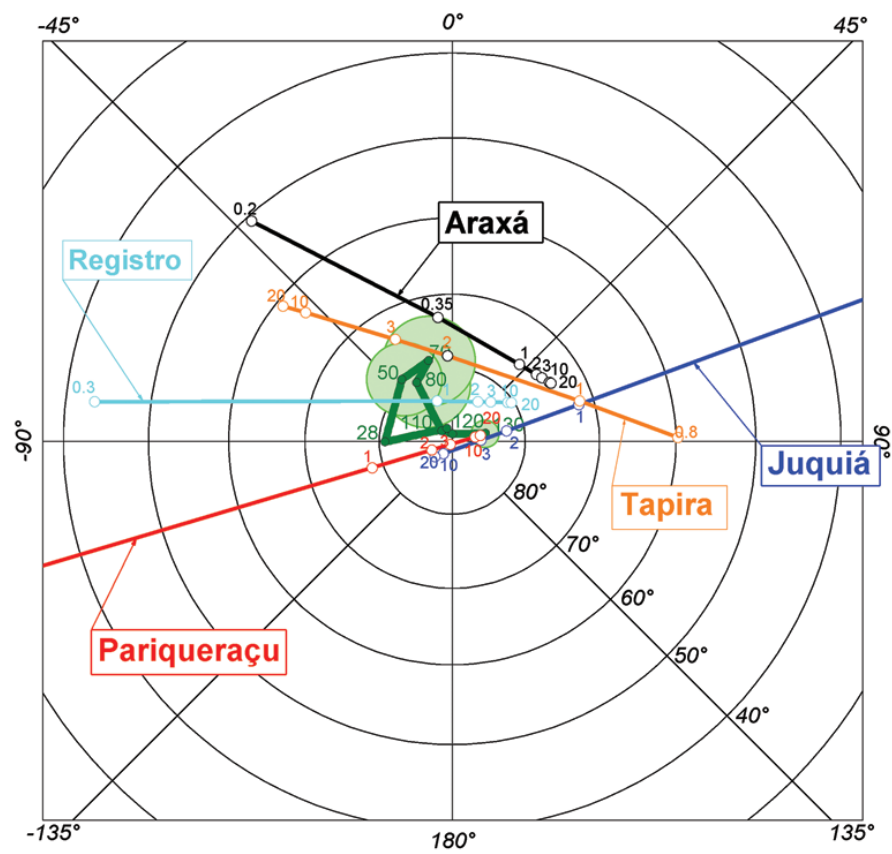

Figura 10 - Curva de deriva polar aparente (APWP) para o Brasil e posição do pólo magnético virtual das anomalias utilizadas, para diferentes valores de $Q$.

plexos alcalinos cuja idade radiométrica é bem conhecida: Araxá, Tapira e Juquiá, e resultados geologicamente coerentes para os outros dois complexos cuja idade da rocha fonte não é conhecida, Pariqueraçu e Registro. Essas estimativas de idade foram efetuadas apenas a partir da anomalia magnética, sem que a rocha tenha sido amostrada.

Entendemos que a magnetização remanescente deve ser considerada como informação, e não como empecilho, na interpretação de anomalias magnéticas brasileiras.

\section{REFERÊNCIAS}

AMARAL G, BUSHEE J, CORDANI UG \& KAWASHITA K. 1967. Potassium-argon ages of alkaline rocks from southern Brazil. Geochimica et Cosmochimica Acta, 31: 117-142.

CORDANI R. 2008. Desenvolvimento de metodologia para aplicar técnicas do paleomagnetismo em anomalias magnetométricas em crosta continental: aplicação a anomalias brasileiras. Instituto de Astronomia, Geofísica e Ciências Atmosféricas - USP. Tese de doutorado, 110 pp. 
CORDANI R \& SHUKOWSKY W. 2009. Virtual Pole from Magnetic Anomaly (VPMA): A procedure to estimate the age of a rock from its magnetic anomaly only. Journal of Applied Geophysics, October, 69(2): 96-102.

ERNESTO M. 2007. Drift of South America Platform since Early Cretaceous: Reviewing the apparent polar wander path. Geociências (São Paulo), 25(1): 83-90.

FEDI M, FLORIO G \& RAPOLLA A. 1994. A method to estimate the total magnetization direction from a distortion analysis of magneticanomalies. Geophys. Prospect, 42(3): 261-274.

GOMES CB, RUBERTI E \& MORBIDELLI L. 1990. Carbonatite complexes from Brazil: A review. J. South Amer. Earth Sci., 3: 51-63.

HARTMANN GA \& PACCA IG. 2009. Time evolution of the South Atlantic Magnetic Anomaly. Anais da Academia Brasileira de Ciências, 81(2): 243-255.

HASUI Y \& CORDANI UG. 1968. Idade Potássio-Argônio de rochas eruptivas Mesozóicas do Oeste Mineiro e sul de Goiás. In: Congresso Brasileiro de Geologia, 22., Belo Horizonte, 1968. Anais, Belo Horizonte, SBG, p. 139-143.

KEATING P \& ZERBO L. 1996. An improved technique for reduction to the pole at low latitudes. Geophysics, 61(1): 131-137.

LIX. 2008. Magnetic reduction-to-the-pole at low latitudes; observations and considerations. Leading Edge, 27(8): 990-1002.
McENROE SA \& BROWN LL. 2000. A closer look at remanence-dominated aeromagnetic anomalies: Rock magnetic properties and magnetic mineralogy of the Russell Belt microcline-sillimanite gneiss, Northwest Adirondack Mountains, New York. Journal of Geophysical Research, 105(B7): 16437-16456.

MORBIDELLI L, GOMES CB, BROTSU P, D'ACQUARICA S, GARBARINO C, RUBERTI E \& TRAVERSA G. 2000. The Pariquera-Açu K-alkaline complex and Southern Brazil lithospheric mantle source characteristics. Journal of Asian Earth Sciences, 18: 129-150.

RAJAGOPALAN S. 2003. Analytic Signal vs. Reduction to Pole: Solutions for Low Magnetic Latitudes. Exploration Geophysics, 34(4): 257262.

ROEST WR \& PILKINGTON M. 1993. Identifying remanent magnetization effects in magnetic data. Geophysics, 58: 653-659.

SCHNETZLER CC \& TAYLOR PT. 1984. Evaluation of an observational method for estimation of remanent magnetization. Geophysics, 49(3): 282-290.

SHURBET DH, KELLER GR \& FRIESS JP. 1976. Remanent magnetization from comparison of gravity and magnetic anomalies. Geophysics, 41(1): $56-61$.

THURSTON JB. 2001. Mapping remanent magnetization using the local phase. Geophysics, 66(4): 1082-1089.

\section{NOTAS SOBRE OS AUTORES}

Renato Cordani é geofísico formado pelo IAG-USP (1993), com mestrado (1997) e doutorado (2008) pela mesma instituição. Atua desde a graduação como geofísico consultor e prestador de serviços técnicos especializados para diversas empresas do setor de exploração mineral, no qual contribuiu para a descoberta e desenvolvimento de diversos depósitos minerais. Trabalha com processamento, interpretação e modelamento de diversos métodos geofísicos, principalmente magnetometria, gravimetria, polarização induzida, gamaespectrometria e EM. Publicou diversos trabalhos em Congressos e Simpósios de geofísica, geologia e mineração. Foi secretário da SBGf (2003-2005), conselheiro da SBGf (2009-2011), secretário da Regional Sul da SBGf (2001-2003), e é revisor da RBG e RBGf.

Wladimir Shukowsky possui graduação em Engenharia de Eletricidade Modalidade Eletrônica pela Universidade de São Paulo (1972), mestrado em Geofísica pela Universidade de São Paulo (1979) e doutorado em Geofísica pela Universidade de São Paulo (1986). Atualmente é Professor Doutor da Universidade de São Paulo, Revisor de periódico da Revista Brasileira de Geociências e Revisor de periódico da Revista Brasileira de Geofísica. Tem experiência na área de Geociências, com ênfase em Geofísica. Atuando principalmente nos seguintes temas: Teoria do potencial, Gravimetria, Litosfera, Problema Inverso, Magnetometria por Satélite. 